\title{
PERDA PRECOCE DE DENTES DECÍDUOS: ETIOLOGIA, EPIDEMIOLOGIA E CONSEQÜÊNCIAS ORTODÔNTICAS
}

\author{
EARLY LOSS OF PRIMARY TEETH: ETIOLOGY, \\ EPIDEMIOLOGY AND ORTHODONTIC CONSEQUENCES
}

\author{
Catarina Ribeiro Barros de Alencar ${ }^{1^{*}}$ Alessandro Leite Cavalcanti ${ }^{1}$, \\ Priscilla Kelly Medeiros Bezerra ${ }^{1}$ \\ $1^{*}$ Autor para contato: Universidade Estadual da Paraíba - UEPB, Departamento de \\ Odontologia, Campina Grande, PB, Brasil. (83) 3333-1393; \\ e-mail: catarinalencar@hotmail.com
}

Recebido para publicação em 08/03/2007

Aceito para publicação em 16/08/2007

\section{RESUMO}

A ocorrência de perdas precoces de dentes decíduos, além de poder ocasionar transtornos comportamentais, repercute fortemente no desenvolvimento da oclusão futura, tendo relação com a fonética e função mastigatória do indivíduo. Sendo assim, torna-se imprescindível que o profissional detecte a presença de uma perda precoce e escolha a conduta clínica mais adequada a cada caso, proporcionando mais segurança e eficácia no tratamento e reabilitação do paciente. Neste cenário, destacam-se os diversos tipos de aparelhos mantenedores e recuperadores de espaço, com indicação variável de acordo com o elemento perdido, idade da criança e características do arco dentário. Objetivamos, pois, rever a literatura sobre as etiologias, complicações ortodônticas futuras, alterações comportamentais comumente observadas em tais pacientes, bem como os tratamentos atualmente empregados com sucesso nessa área.

Palavras-chave: perda de dente; dente decíduo; mantenedor de espaço

\begin{abstract}
The occurrence of early loss of deciduous teeth, besides the possibility of causing behavior disturbances, strongly reflects on the development of future occlusion, bearing direct relation with the individual's phonetic and chewing functions. Thus, the dentist must be able to detect the presence of an early loss and to make the appropriate choice for the best clinical procedure in each case, providing more security and effectiveness in the treatment and rehabilitation of the
\end{abstract}


patient. In such cases, the dentist can choose among various types of space maintainers, according to the lost element, the age of the child and characteristics of the dental arch. So, our aim is to review the literature on the etiologies, orthodontic complications and behavior alterations observed in such patients, as well as the current treatments successfully used in this area.

Key words: tooth loss, deciduous tooth, space maintenance

\section{Introdução}

As perdas precoces de dentes decíduos têm sido frequentemente destacadas em estudos epidemiológicos (Alamoudi, 1999; Alsheneifi e Hughes, 2001; Batista, 2006; Cardoso et al., 2005; Gasparim et al., 2003; Martinez et al., 2005; Nogueira et al., 1998; Thomaz et al., 2002) frente a sua relevância e associação com o surgimento de anormalidades de oclusão (Dearing, 1994; Guedes-Pinto, 1991; Krakowiak, 1966; Pedersen et al., 1978; Rose, 1966).

A dentição humana compreende duas fases: decídua ou temporária e permanente, sendo que em determinada etapa do desenvolvimento da criança (entre 6 e 12 anos de idade) dentes decíduos e permanentes estão presentes na cavidade bucal, constituindo a dentição mista (Sucupira, 2000).

Segundo Nogueira et al. (1998) o dente decíduo é importante para o bom desempenho das funções mastigatória, articulação, fonação e oclusão. Long (1999) acrescentou ainda a participação de tais elementos durante os períodos do crescimento e desenvolvimento da altura dos arcos dentais, na respiração e na harmonia estética do corpo da criança, sendo assim, fundamental a sua manutenção até a época normal de esfoliação.

Além disso, a dentição decídua é importante para o desenvolvimento dos maxilares e músculos da face, servindo de guia para os dentes permanentes irromperem em posição correta. (Pinto, 2000).

Garcia et al. (2003) destacaram que a deterioração dos dentes decíduos além de interferir na função mastigatória pode influenciar no crescimento corporal e craniofacial das crianças.

A literatura apresenta várias definições para o termo "perda precoce". A perda de um dente decíduo é considerada precoce ou prematura quando ocorre antes do tempo de sua esfoliação normal (Brusola, 1989). Moyers (1991) determina que a perda precoce ocorre quando um dente decíduo é perdido antes do sucessor permanente ter começado a sua erupção (formação coronária completa e formação radicular já iniciada). Para Araújo (2002), essa perda pode ser considerada precoce quando ocorre com um espaço de tempo, de pelo menos, um ano antes da erupção do sucessor permanente.

O problema maior surge quando a perda de um dente decíduo se processa um pouco antes da raiz do elemento sucessor permanente estar formada (entre os estágios 5 e 6 de Nolla). Nestes casos forma-se uma espécie de fibrose, chamada cicatricial, que parece atrasar a erupção do dente permanente, com conseqüente deslocamento dos dentes adjacentes e extrusão do dente antagonista (Garcia, 2003).

Segundo Guedes-Pinto (1991) a perda prematura de molares decíduos determina a erupção precoce de dentes permanentes, caso ocorra num período mais ou menos próximo da sua esfoliação normal, e um atraso no período de erupção dos seus sucessores se esta ocorrer numa época muito precoce ao de sua esfoliação normal, isso porque no local da perda ocorre neoformação óssea sobre o germe dental, além da fibrose no tecido gengival devido ao traumatismo causado pela mastigação.

Logo, a perda precoce de dentes decíduos além de resultar em problemas estéticos, ortodônticos e fonéticos, pode alterar a relação entre os maxilares e a criança poderá desenvolver hábitos nocivos de postura de língua que também comprometem a fonação (Coelho, 2003).

O presente trabalho objetiva revisar a literatura sobre a perda precoce de dentes decíduos, destacando 
aspectos relacionados à etiologia, epidemiologia, alterações oclusais pertinentes, bem como as alterações comportamentais dos pacientes e as condutas terapêuticas atualmente utilizadas.

\section{Revisão de Literatura Etiologia}

Traumatismo dental, reabsorção prematura das raízes dentárias e cárie são apontadas como as principais causas responsáveis pela perda precoce de dentes decíduos. (Martins, 1998).

Pinkhan (1995) elucidou que na dentição decídua a perda precoce pode ocorrer nos dentes anteriores e posteriores. Com relação à perda dental anterior, principalmente de incisivos, sua etiologia está relaciona ao traumatismo e a cárie dentária. Em dentes posteriores, a maior parte das perdas precoces ocorre por lesões de cárie.

Os traumatismos dentários são comuns na dentição decídua, principalmente na faixa entre 1 e 3 anos de idade, com maior freqüência os dentes anteriores. (Andreasen, 1970). Nesta faixa etária, a criança aprende a andar e depois correr (Christensen e Felds, 1996), ainda sem coordenação motora e capacidade de decisão completamente desenvolvidas, portanto, mais sujeita à quedas. (Fried et al., 1996)

A reabsorção prematura das raízes dentárias ocorre antes da época normal de esfoliação e pode estar presente em arcos dentários apinhados, sendo encontrada principalmente nos incisivos laterais e caninos decíduos inferiores e superiores (Van Der Linden, 1986). Segundo Martins (1998) a reabsorção prematura pode ocorrer devido à erupção de um permanente que entre em contato com a proeminência da superfície de um dente decíduo causando reabsorção radicular atípica nessa região.

Segundo Tomita et al. (2000) as crianças brasileiras apresentam altos índices de extrações dentárias sem a devida manutenção do espaço perdido. Estas ocorrem principalmente devido a presença de extensas lesões de cárie, que, de acordo com Araújo (2002) é a doença que mais atinge a dentição, causando desconforto, dor, e limitações ao indivíduo que a possuem.

Assim sendo, a extração precoce de dentes decí- duos repercute diretamente na desarmonia entre os arcos dentários, prejudicando o desenvolvimento da oclusão futura.

\section{Epidemiologia e distribuição}

Nogueira et al. (1998) ao analisarem a perda precoce na cidade de Belém em 1.830 pacientes, observaram que 280 deles $(15,3 \%)$ apresentaram perda precoce de um ou mais dentes. A maior prevalência de perda foi dos molares decíduos (49,8\%), dos quais o primeiro molar inferior decíduo apresentou maior freqüência de perda $(12,6 \%)$. Constataram também que as principais causas da perda precoce de dentes foram a cárie dentária e o trauma.

Alamoudi (1999) avaliou a prevalência de perda precoce de molares decíduos e apinhamento na dentição decídua. O exame realizado em 502 crianças entre 4 e 6 anos, detectou que 31 crianças $(6,2 \%)$ apresentaram perda prematura de dentes decíduos. Concluiu que o dente mais comumente perdido foi o elemento 84 e que no geral as perdas de primeiro molar foram significativamente maiores que as de segundo molar.

Alsheneifi e Hughes (2001) constataram, após a análise de 277 prontuários de pacientes na faixa etária de 3 a 13 anos que tiveram pelo menos um dente decíduo extraído, que as principais causas das exodontias foram as lesões de cáries decorrentes de patologias pulpares, com o primeiro molar decíduo o elemento que apresentou o maior índice de extração precoce.

Thomaz et al. (2002) avaliaram, por inspeção visual, a perda precoce em 989 crianças de 2 a 5 anos de idade matriculadas em creches das cidades de Aracajú (SE), Bayeux (PB), João Pessoa (PB) e Recife (PE). A perda prematura de dentes decíduos encontrada na amostra foi de $2,9 \%$ sem diferença estatisticamente significativa entre os gêneros. Do total de perdas constatadas (40 elementos perdidos), a maioria decorreu de trauma na região dos incisivos superiores, $22(55 \%)$.

Gasparim et al. (2003) em um estudo epidemiológico sobre as condições bucais e a prevalência de maloclusão em escolares de 6 a 8 anos de idade da cidade de Curitiba concluíram que de um total de 1279 crianças, 68 apresentavam perdas prematuras, 121 apresentavam destruições extensas e 68 apresentavam 
destruições extensas e perdas prematuras concomitantes. As crianças portadoras de maloclusão de Classe I apresentaram os maiores índices de perdas prematuras de dentes.

Cardoso et al. (2005) avaliaram a prevalência de perdas precoces de primeiros e segundos molares decíduos de crianças atendidas na disciplina de odontopediatria da Universidade Luterana do Brasil, Torres/ RS, e constataram através da análise de 404 prontuários de pacientes atendidos entre março de 2001 e julho de 2002, que 172 deles apresentaram perda precoce de molares decíduos (prevalência de 42,6\%). A cárie dentária representou $100 \%$ da causa de perdas precoces e os dentes mais comumente perdidos foram os segundos molares decíduos inferiores.

Martinez et al. (2005) desenvolveram um estudo transversal com 480 crianças entre 5 e 10 anos de idade, de ambos os gêneros, no período compreendido entre janeiro e maio de 2003, em Holguín, Cuba. Observaram que $18,54 \%$ das crianças tinham perdas prematuras, sendo o primeiro molar decíduo o dente mais afetado e, aproximadamente, metade das crianças com perdas precoces apresentaram algum efeito consecutivo associado à maloclusão dentária.

Batista (2006) estudou a prevalência e etiologia da perda precoce de dentes decíduos nos pacientes atendidos na clinica de odontopediatria da Universidade Federal de Santa Catarina através da análise de 500 prontuários, perfazendo um total de 762 exodontias. Desse total, 326 casos $(42,8 \%)$ corresponderam à perda fisiológica e 436 casos $(57,2 \%)$ corresponderam à perda precoce de dentes decíduos, sendo que destes $16,5 \%$ eram dentes anteriores e $83,5 \%$ eram posteriores. Os dentes com maior prevalência de perdas precoces foram o segundo molar inferior direito $(14,5 \%)$ e o primeiro molar inferior direito $(12,8 \%)$. Os principais fatores etiológicos das perdas prematuras foram a cárie $(35,1 \%)$ e a reabsorção patológica das raízes $(16,5 \%)$. A comparação da prevalência de perdas de elementos mandibulares e maxilares revelou maior ocorrência de perdas no arco inferior (50,9\%).

\section{Conseqüências ortodônticas das perdas precoces de dentes decíduos}

As seqüelas das perdas precoces dependem do dente perdido, da idade que tinha o paciente quando ocorreu a perda, do espaço disponível na arcada dentária, do padrão de erupção e de intercuspidação, da função muscular, das características esqueléticas individuais e das condições periodontais (Kisling e Hoffding, 1979).

Embora grande parte dos autores descreva a perda precoce como fator etiológico direto das maloclusões (Dearing, 1994; Guedes-Pinto, 1991; Krakowiak, 1966; Van Der Linder, 1986), alguns chegaram a analisá-la com pouca interferência no surgimento de anormalidades de oclusão, relação de $8 \%$ para o estudo de Willet (1931) e mesmo nenhuma interferência, nos levantamentos de Inoue (1983).

Porém, em grande parte dos casos, as perdas contribuem para a ruptura do equilíbrio dentário, acarretando marcas irreversíveis no germe do dente sucessor permanente, na oclusão do indivíduo e desarmonia do sistema estomatognático como um todo (Proffit, 1978).

Ademais, Kronfeld (1953) afirma que quanto maior é o intervalo entre a perda precoce e a erupção do seu sucessor permanente, maior será a probabilidade de má posição do permanente.

A perda precoce de dentes decíduos resultante de cáries e traumatismos traz como conseqüência imediata um grande número potencial de distúrbios diretos e indiretos da oclusão, entre os mais comuns temos perda de espaço, extrusão de dente antagonista, torsiversão de dentes adjacentes e problemas adicionais como deglutição atípica (Guedes-Pinto, 1991).

Em muitos casos, a perda precoce pode acarretar o mau posicionamento dos dentes adjacentes e pode influenciar até mesmo no padrão de erupção dos segundos molares permanentes, por não promover o crescimento correto das bases ósseas (Krakowiak, 1966).

O surgimento de sobreerupção do dente antagonista e desvio de linha média decorrida de uma perda precoce são relatados por Rose (1966), além de impacção dos dentes sucessores devido a movimentação dos dentes vizinhos em direção ao dente perdido (Dearing, 1994).

Constata-se também, depois de ocorrida uma perda prematura, encurtamento do arco dentário (Van Der Linden, 1986). 
Em um estudo longitudinal sobre modificações ocorridas no arco após a perda prematura de primeiros molares decíduos, Cuoghi et al. (1998) concluíram que a extração precoce de primeiro molar decíduo durante a fase da dentição mista promove uma redução de espaço com o deslocamento dos dentes permanentes.

Rao e Sarkar (1999) estudaram as modificações no arco após perdas precoces de molares decíduos verificando, entretanto, que pode ocorrer redução ou aumento no comprimento do arco e que a redução deve-se à migração mesial do molar e o aumento decorre da migração distal do canino.

A influência no local da extração foi analisada por Pedersen et al. (1978) em um estudo sobre a freqüência de maloclusão em um grupo de crianças com perdas na mandíbula ou maxila isoladas. Constatou-se que a perda precoce de dentes decíduos resultou no aumento da freqüência de maloclusão sagital, vertical e transversal, notou-se também, que diante da gravidade dos casos, as extrações na mandíbula repercutem na necessidade de tratamentos ortodônticos mais prolongados.

Breakspear(1951) relatou que o fechamento de espaço é mais rápido e mais completo na perda do segundo molar decíduo, do que no primeiro molar decíduo, sendo que a migração é maior na maxila se comparada à mandíbula.

\section{Alterações comportamentais observadas em crianças após a perda prematura de dentes decíduos}

A importância estética dos dentes temporários anteriores é assertiva. A normalidade da forma, coloração e do posicionamento dos dentes dá lugar a uma harmonia que influência positivamente o desenvolvimento da auto-estima da criança (Garcia, 2003). Portanto, a perda precoce de dentes anteriores pode ter um impacto profundo na auto-estima das pessoas (Rodd e Atkin, 2000). Em crianças, pode gerar problemas de comportamento, vinculados a sentimentos de depressão e isolamento (Piassi et al., 2000).

Os defeitos estéticos dos dentes pioram a atitude da criança com relação a si mesma e aos outros, principalmente se esses defeitos são visíveis durante a fala e o riso (Moreira, 1993).
Dessa forma, uma disposição deprimida pode diminuir o nível de energia, podendo afetar o desempenho da criança na escola e em outros lugares, levando a uma diminuição continua da auto-estima, sendo freqüente a preservação do autoconceito negativo muito tempo após o termino da infância (Papalia e Olds, 2000).

Portanto, a perda precoce de dentes decíduos, com subseqüente comprometimento estético pode induzir alterações comportamentais e repercussões no ajuste social, fatores que devem ser considerados no planejamento do tratamento (Damasceno et al., 2002).

\section{Manutenção de espaço}

Atualmente vários dispositivos podem ser utilizados para manutenção de espaço após a perda precoce de um dente decíduo, dentre os quais se encontram: Banda-alça, Coroa-alça, Arco lingual de Nance e Botão palatino de Nance.

Duas situações podem ocorrer após a perda precoce de um dente decíduo: o espaço correspondente ao dente decíduo perdido encontra-se preservado, havendo a possibilidade de movimentação de dentes adjacentes e conseqüente perda deste espaço, ou o espaço já se encontra comprometido com a inclinação mesio-distal de dentes adjacentes. No primeiro caso utilizam-se os aparelhos mantenedores de espaço e no segundo, faz-se a reabilitação do arco através do uso de aparelhos recuperadores de espaço.

Contudo, para a manutenção de espaço, nem sempre é necessário o uso de aparelhos ortodônticos. Dolci e Ferreira (2003) evidenciam que em casos de cáries proximais ou dentes anquilosados, por exemplo, a manutenção do espaço pode ser conseguida através de uma restauração adequada.

Os mantenedores de espaço são dispositivos ortodônticos que substituem um ou mais dentes e têm sido utilizados para preservar o espaço destinado ao elemento dentário permanente sucessor, sem que este se torne impactado ou sofra desvios durante sua erupção (Diniz et al., 2005).

Os aparelhos mantenedores de espaço são classificados quanto a sua funcionalidade em funcionais $\mathrm{e}$ não funcionais, e quanto a fixação, em fixos e removíveis. 
Aparelhos com a característica de reestabelecimento da funcionalidade do dente perdido são especialmente indicados em casos de maior probabilidade de ocorrência de perda de espaço no sentido vertical, além do mesio-distal. Apresentam a vantagem de devolver a função mastigatória e evitar a extrusão do dente antagonista.

Bijoor e Kohli (2005) afirmam que estes aparelhos não preservam somente a função e o comprimento do arco, mantêm também a estética e eliminam os danos psicológicos que uma criança poderia enfrentar em conseqüência da perda prematura dos dentes.

Os aparelhos removíveis apesar de apresentarem a vantagem de evitar a confecção de mais de um aparelho, conforme a modificação dimensional do arco, necessitando apenas de alguns ajustes segundo a erupção dentária, apresentam o grande inconveniente de depender diretamente da colaboração do paciente com o tratamento. Esses aparelhos são confeccionados em resina acrílica, com a possibilidade de inclusão de dentes artificiais, estando mais indicados em casos de perdas anteriores e perdas múltiplas de dentes.

Os aparelhos fixos exigem maiores cuidados tanto do profissional quanto do paciente. São confeccionados em material metálico, e os seus principais tipos são assim descritos por Paixão e Fuziy (2003):

a) Banda-alça: é um aparelho indicado em casos que o espaço da perda precoce não é muito extenso, principalmente em perda unilateral de primeiro e segundo molar decíduo com primeiro molar permanente presente, no qual se faz a adaptação da banda ortodôntica que sustentará o aparelho.

b) Coroa- alça: tem a mesma indicação do aparelho tipo Banda-alça, sendo utilizado em casos particulares de deterioração do dente suporte por cáries extensas. A coroa metálica tem a função de conferir maior resistência ao conjunto.

c) Arco lingual de Nance: é um aparelho indicado em casos de perdas prematuras bilaterais de molares decíduos, com melhor aplicabilidade quando os primeiros molares permanentes já estiverem irrompidos.

d) Botão palatino de Nance: é um aparelho indicado em casos de perdas múltiplas e bilaterais de molares decíduos superiores e assim como os demais aparelhos fixos, as bandas ortodônticas são assentadas nos primeiros molares permanentes.
Os recuperadores de espaço são dispositivos dinâmicos, cuja finalidade é recuperar espaço nos casos em que o diâmetro mesiodistal, destinado ao dente permanente em fase de erupção, foi perdido parcialmente (Guedes-Pinto, 1991). Podem ser fixos ou removíveis, podendo-se empregar a ancoragem extrabucal ou a placa lábio-ativa. A primeira, utilizada principalmente em dentições mistas, serve para recuperar ou ganhar espaço pela distalização dos primeiros molares permanentes superiores; a segunda, é composta por um fio de aço adaptado aos tubos das bandas dos primeiros molares permanentes, permitindo a verticalização destes e a recuperação do comprimento do arco.

\section{Discussão}

Diante da importância da dentição decídua não só para o desenvolvimento satisfatório da dentição permanente, mas também para o desempenho das funções mastigatória e fonética, tendo relação com a articulação e oclusão do indivíduo, a literatura converge para a importância da manutenção do dente decíduo até a época de sua esfoliação natural.

Garcia (2003) considera apenas a possibilidade da perda precoce levar a um atraso na erupção do sucessor permanente, enquanto Guedes-Pinto (1991) analisa a perda precoce como uma situação que, dependendo do tempo em que ocorre, pode atrasar ou acelerar a processo de erupção do dente permanente.

Enquanto alguns estudos revelam profunda relação da perda precoce com a maloclusão, citandose principalmente perda de espaço, extrusão de dente antagonista, torsiversão de dentes adjacentes (GuedesPinto, 1991), mau posicionamento dos dentes adjacentes e influência no padrão de erupção dos segundos molares permanentes (Krakowiak, 1966), sobreerupção do dente antagonista e desvio de linha média (Rose, 1966), impacção dos dentes sucessores (Dearing, 1994), e encurtamento do arco dentário (Van Der Linden, 1986), alguns achados epidemiológicos como os de Inoue (1983) não encontraram relação positiva entre as variáveis descritas.

No que se refere ao fator etiológico, a cárie foi apontada na maior parte dos estudos como fator etiológico principal das perdas precoces (Nogueira et al., 
1998; Cardoso et al., 2005; Batista, 2006; Alsheneifi e Hughes, 2001) outros fatores citados como secundários foram o trauma no estudo de Nogueira (1998) e a reabsorção patológica de raízes no estudo de Batista (2006).

Quanto ao elemento mais acometido por perda precoce encontrou-se divergência de resultados de um estudo para outro. Nogueira et al. (1998) encontraram maior freqüência de perda para o primeiro molar inferior decíduo. $\mathrm{O}$ primeiro molar também foi mais acometido que o segundo, nos estudos de Alamoudi (1999) e Martinez et al. (2005). Cardoso et al. (2005) verificaram, entretanto, que os segundos molares decíduos inferiores são os dentes mais acometidos por perda precoce. Posteriormente, Batista (2006) encontrou maior prevalência de perda para o segundo molar inferior direito. Thomaz et al. (2002), contudo obtiveram dados cuja análise permitiu afirmar que os incisivos superiores tiveram maiores freqüências de perda do que os molares. As divergências de resultados provavelmente são explicadas pela diferença de características das amostras estudadas.

Quanto as alterações comportamentais observadas em crianças após a perda prematura de dentes decíduos, os autores (Garcia, 2003; Rodd e Atkin, 2000; Piassi et al., 2000; Moreira, 1993; Papalia e Olds, 2000; Damasceno et al., 2002) são unânimes em afirmar que a perda de um dente decíduo antes da época normal leva a danos psicológicos consideráveis que refletem a insatisfação do indivíduo consigo mesmo e o sentimento de inferioridade diante das demais crianças. Logo, a devolução da função estética, em alguns casos severamente prejudicada, deve ser considerada pelo profissional.

No que concerne à relação entre perda precoce e maloclusão, Durward e Ann (2000) afirmam que a perda precoce pode resultar em diminuição do arco dentário conduzindo à maloclusão. Os mantenedores de espaço são citados pelos autores como dispositivos que frequentemente podem prevenir a perda de espaço e, além disso, prevenir o desenvolvimento de uma maloclusão futura ou reduzi-la severamente. A importância do uso de tais dispositivos nos casos de perdas precoces é ressaltada ainda nos estudos de Diniz et al. (2005), Bijoor e Kohli (2005) e Paixão e Fuziy (2003) como medida capaz de amenizar as alterações na fonética e atividade mastigatória do individuo.

\section{Conclusões}

A situação da saúde bucal das crianças brasileiras é preocupante e os índices de perdas precoces sem a devida manutenção de espaço são elevados. Ressaltase, portanto a importância de medidas que contribuam para a manutenção e reestabelecimento não só da função mastigatória, mas também da estética, fonética e dos aspectos psicológicos frequentemente perturbados decorrida a perda de um elemento dentário. Assim sendo, é dever do cirurgião-dentista, principalmente do odontopediatra, zelar pela integridade da dentição decídua frente a sua importância para o íntegro desenvolvimento da dentição permanente, contribuindo, assim, para o equilíbrio geral do sistema estomatognático do indivíduo.

\section{REFERÊNCIAS}

1. ALAMOUDI, N. The prevalence of crowding, attrition, midline discrepancies and premature tooth loss in the primary dentition of children in Jeddah, Saudi arabia. J Clin Pediatr Dent, Birmingham, v.24, n.1, p. 53-58, Fall, 1999.

2. ALSHENEIFI, T; HUGHES, C. V. Reasons for dental extractions in children. Pediatr Dent, Chicago, v. 23, n. 2, p. 109-112, Mar./Apr. 2001.

3. ANDREASEN, J. O. Etiology and pathogenesis of traumatic dental injuries. A clinical study of 1,298 cases. Scand J Dent Res, Copenhagen, v. 78, n. 4, p. 329-342, 1970.

4. ARAUJO, F. M. Relação entre o tipo de aleitamento e o uso de chupeta. J Bras Odontopediatr Odontol Bebê, Curitiba, v.3, n. 25, p. 235-240, mai./jun. 2002.

5. BATISTA, A. M. R. Prevalência e etiologia da perda precoce de dentes decíduos nos pacientes atendidos na clinica de odontopediatria da Universidade Federal de Santa Catarina. 2006. 126 f. Dissertação (Mestrado, Universidade Federal de Santa Catarina, Florianópolis, 2006.

6. BIJOOR, R. R; KOHLI, K. Contemporary space maintenance for the pediatric patient. N Y State Dent J, New York, v.71, n.2, p. 32-35, Mar. 2005.

7. BREAKSPEAR, E. K. Sequelae of early loss of deciduous molars. Dent Rec, London, v.71, n.7, p. 127-135, Jul./Aug. 1951.

8. BRUSOLA, J.A.C. Ortodontia clínica. Barcelona: Sabat, 1989. $506 \mathrm{p}$. 
9. CARDOSO, L. et al. Avaliação da prevalência de perdas precoces de molares decíduos. Pesq Bras Odontoped Clin Integr, João Pessoa, v.5, n.1, p. 17-22, jan./abr. 2005.

10. CHRISTENSEN, J. R; FELDES, H. W. Manutenção de espaço na dentição decídua. In: PINKHAM, J. R. Odonto-pediatria da infância a adolescência. 2. ed. São Paulo: Artes Médicas, 1996, p. 392-399.

11. COELHO, M. L. G. Perda precoce da dentição decídua: análise da percepção das mães de crianças de 02 a 06 anos de idade na sede do distrito de Jaibaras, Sobral - CE. 54f. Monografia (Especialização), Universidade Estadual do Vale do Acaraú, Sobral, 2003.

12. CUOGHI, O. A. et al. Loss of space and dental arch length after the loss of the lower first primary molar: a longitudinal study. J Clin Pediatr Dent, Birmigham, v.22, n.2, p.117-120, Winter, 1998.

13. DAMASCENO, L. et al. Alterações no comportamento infantil decorrente da perda precoce de dentes anteriores: relato de caso. RBO, Rio de Janeiro, v. 59, n. 3, p. 123-126, mai./jun. 2002.

14. DEARING, S. G. Space loss and malocclusion. N Z Dent J, Dunedin, v. 44, n. 4, p. 365-370, Apr. 1994.

15. DINIZ, M. B. et al. Perda dental precoce e manutenção de espaço na dentadura decídua - Relato de um caso clínico. J Bras Odontopediatr Odontol Bebê, Curitiba, v. 8, n. 44, p. 376381, 2005.

16. DOLCI, G. S; FERREIRA, E. J. B. Tratamento ortodôntico preventivo e interceptativo da perda precoce de dentes decíduos: manutenção de espaço. Rev Odonto Ciência, Porto Alegre, v.18, n. 41, p. 290-298, jul./set. 2003.

17. DURWARD, C. S; ANN R. Space maintenance in the primary and mixed dentition. Ann Australas Coll Dent Surg, Sydney, v.15.p. 203-205, Oct. 2000.

18. FRIED, I. et al. Subluxation injuries of maxillary primary anterior teeth: epidemiology and prognosis of 207 traumatized teeth. Pediatr Dent, Chicago, v.18, n. 2, p. 145-151, Mar./Apr. 1996.

19. GARCIA I. F. et al. Importancia de los dientes temporales. Su cronología de erupcion. Rev Pediatr Aten Primaria, Barcelona, v. 5, p. 439-445, 2003.

20. GASPARIM, G. R. et al. Levantamento epidemiológico da prevalência de maloclusões e das condições bucais em escolares da cidade de Curitiba. Revista Odonto Ciência, Porto Alegre, v.18, n.42, p. 373-376, out./dez. 2003.

21. GUEDES-PINTO, A. C. Odontopediatria. 3. ed. São Paulo: Santos, 1991. 1126p.

22. INOUE, N. et al. Influence of tooth-to-denture-base discrepancy on space closure following premature loss of deciduous teeth. Am J Orthod, St. Louis, v.83, n.5, p. 428-434,
May, 1983.

23. KISLING, E; HOFFDING, J. Premature loss of primary teeth: art III, drifting patterns for different types of teeth loss of adjoining teeth. J Dent Child, Chicago, v. 46, p. 34-38, Jan./ Feb. 1979 .

24. KRAKOWIAK, F. J. Growth potential of mandible as factor in mesial movement of permanent first molar. J Dent Child, Chicago, v. 31, n. 4, p. 278, Sep. 1966.

25. KRONFELD, S. M. the effects of premature loss of primary teeth and sequence of eruption of permanent teeth of malocclusion. J Dent Child, Chicago, v. 20, p. 2-13, 1953.

26. LONG, S. M Análise morfológica da dentição decídua. $90 f$. Tese (Doutorado em Odontopediatria), Faculdade de Odontologia, Universidade de São Paulo, São Paulo, 1999.

27. MARTÍNEZ, N. S. et al. Pérdida prematura de dientes temporales y maloclusión en escolares. Policlínica "Pedro Díaz Coello", 2003. Disponível em: http: www.cocmed.sld.cu/no93/ n93ori4.htm. Acesso em: 10 Dez 2005.

28. MARTINS, A. L. C. F. Odontopediatria na $1^{a}$ infância. São Paulo: Santos, 1998. Cap. 17.

29. MOYERS, R. E. Etiologia da maloclusao. In: Ortodontia. Rio de Janeiro: Guanabara-Koogan, 1991.p. 127-140.

30. MOREIRA, S. C. Prótese dentária - prótese fixa para bebês. ROBRAC, Goiânia, v.3, n.8, p.26, jul./set. 1993.

31. NOGUEIRA, A. J. S. et al. Perdas precoces de dentes decíduos e suas conseqüências para dentição futura - elaboração de propostas preventivas. Rev ABO Nac, v.6, n.4, p. 228-233, 1998.

32. PAIXAO, R. F.; FUZIY,A. Uma abordagem ortodôntica das perdas dentais precoces. In: $15^{\circ}$ Conclave Odontológico de Campinas. Campinas, n. 104, p. 1678-1899, 2003.

33. PAPALIA, D. E.; OLDS, S. W. Desenvolvimento humano. 7. ed. Porto Alegre: Artes Médicas, 2000.

34. PEDERSEN, J. et al. Prevalence of malocclusion in relation to premature loss of primary teeth. Community Dent Oral Epidemiol, Copenhagen, v. 6, n. 4, p. 204-209, Jul. 1978.

35. PIASSI, E. et al. Mantenedor de espaço fixo para região anterior na dentição decídua. J Bras Odontopediatr Odontol Bebê, Curitiba, v.3, n.14, p. 237-278, jul./ago. 2000.

36. PINKHAM, J. R. Odontopediatria da infância à adolescência. 2. ed. São Paulo: Artes Médicas, 1995. p. 393415.

37. PINTO, V. Prevenção da carie dental. In: Saúde bucal coletiva. 4. ed. São Paulo: Santos, 2000. p. 371-384.

38. PROFFIT, W. R. Equilibrium theory revisited. Factors influencing position of the teeth. Angle Orthod, Appleton, v.48, n.3, p. $175-186,1978$. 
39. RAO, A. K; SARKAR S. Changes in the arch length following premature loss of deciduous molars. J Indian Soc Pedod Prev Dent, Chandigarh, v. 17, n.1, p.29-32, Mar. 1999.

40. RODD, H. D; ATKIN, J. M. Denture satisfaction and clinical performance in a pediatric population. Int J Paediatr Dent, Oxford, v.10, p. 27-37, 2000.

41. ROSE, J. S. Early loss of teeth in children. Br Dent J, London, v 120, n. 6, p. 275-280, Mar. 1966.

42. SUCUPIRA, A. C. et al. Pediatria em consultório: Saúde e as doenças bucais. 4. ed. São Paulo: Saraiva, 2000. p. 48-60.

43. TOMITA, N. E. et al. Relação entre hábitos bucais e má oclusão em pré-escolares. Rev Saúde Pública, São Paulo, v.34, n.3, p.299-303, jun. 2000.

44. THOMAZ, E. B. A. F. et al. Prevalência de protrusão dos incisivos superiores, sobremordida profunda, perda prematura de elementos denatários e apinhamento na dentição decídua. J Bras Odontopediatr Odontol Bebê, Curitiba, v.5, n.26, p. 276-282, jul./ago. 2002.

45. VAN DER LINDEN, F. P. G. As conseqüências das perdas prematuras dos dentes decíduos. In:

Ortodontia:

Desenvolvimento da dentição. São Paulo: Quintessence, 1986. p. 129-153.

46. WILLET, R. C. Children dentistry for on orthodontics pointol. J Orthod, Oxford, v. 17, p.552-565, 1931. 